\title{
Sémantique des jugements épistémiques : degré de croyance et prise en charge
}

\section{On epistemic statements: degree of belief and commitment}

\author{
Laurent Gosselin \\ Université de Rouen \\ DySoLa, EA 4701
}

laurent.gosselin@univ-rouen.fr

IRED,

1, rue Thomas Becket,

76821 Mont-Saint-Aignan Cedex

France

Cet article propose une nouvelle analyse des jugements épistémiques (X croire / savoir / s'imaginer que $\mathrm{p}$ ) dans le cadre de la Théorie Modulaire des Modalités (Gosselin 2010). Après avoir critiqué l'application à la sémantique linguistique de certains principes de la logique épistémique (comme celui de l'objectivité du savoir), nous proposons une analyse énonciative de la factivité, qui se trouve nettement distinguée à la fois du degré de croyance et de la factualité. Nous traitons dans ce cadre de la factivité dans le discours rapporté et de la projection des présuppositions. Nous montrons enfin que cette analyse syntactico-sémantique de la modalité peut servir de base à une approche pragmatique de la modalisation dans le discours.

Mots-clefs : modalité épistémique, logique épistémique, factivité, présupposition

In this paper, we propose a new analysis of epistemic statements (X croire / savoir / s'imaginer que $\mathrm{p}$ ) in the framework of the Modular Theory of Modalities (Gosselin 2010). First, we criticize the use, in the field of linguistic semantics, of some principles of epistemic logic (as the principle of objectivity of knowledge). Then, we argue in favor of an enunciative approach of factivity, which is clearly distinguished both from the belief's degree and from factuality. In this framework, we deal with classical problems of factivity in reported speech and of projection of presuppositions. Finally, we show that this semantic analysis of modality can be taken as a base for a pragmatic approach of modalization in discourse.

Key words: epistemic modalities, epistemic logic, factivity, presupposition

\section{Introduction}

Un jugement épistémique exprime une croyance ou un savoir portant sur une proposition. $\mathrm{Ce}$ type de jugement peut rester implicite dans le cas de l'assertion simple (en assertant «p », le locuteur se présente comme croyant/sachant que $\mathrm{p}$ ), ou se trouver explicité et précisé par divers types de marqueurs : adverbes (peut-être, sans doute), coverbes modaux et évidentiels ${ }^{1}$ (pouvoir, devoir), verbes d'attitude propositionnelle (croire, savoir, être certain, se figurer $q u e \mathrm{p}$ ), etc. Cet article propose une analyse de ces verbes épistémiques, centrée sur deux dimensions de la modalité exprimée : a) le degré de croyance du sujet de l'énoncé, et b) la prise en charge de la proposition par le locuteur (sujet de l'énonciation). Soit les exemples :

(1) a. Pierre se doute que Marie est venue

b. Pierre est certain que Marie est venue.

En (1a), le locuteur prend en charge p, à titre de présupposé (le verbe est factif), alors que le sujet de l'énoncé (Pierre) n'entretient qu'une croyance faiblement positive à son égard (Ducrot 1972: 268). En (1b), c'est exactement l'inverse la croyance est forte, mais la proposition n'est pas prise en charge par le locuteur (le prédicat n'est pas factif).

Un moyen de faire apparaître clairement ces distinctions consiste à examiner les inférences valides. De (1a), on infère légitimement «p », alors que ce n'est pas possible pour (1b). Or la logique épistémique s'est donné pour objectif de rendre compte au moyen de

\footnotetext{
${ }^{1}$ Sur le statut modal et/ou évidentiel des coverbes épistémiques, voir Barbet \& de Saussure (éds, 2012).
} 
principes généraux et explicites de l'ensemble des inférences valides que l'on peut obtenir à partir de l'emploi des prédicats épistémiques, et, par cette voie logico-référentielle, des différences sémantiques entre ces prédicats. A la suite d'une discussion critique des résultats obtenus par la logique épistémique, nous proposerons une nouvelle modélisation de ces phénomènes dans le cadre de la Théorie Modulaire de Modalités (Gosselin 2010).

\section{Logique épistémique et sémantique linguistique}

La logique épistémique (cf. Hintikka 1962, Gardies 1979, Lenzen 2004) a été appliquée avec succès à l'analyse linguistique des constructions en «savoir / croire que p » en français par Martin (1987). L'intérêt du recours à la logique épistémique est triple : elle procure un métalangage rigoureux à l'analyse sémantique; elle permet de prédire, au moyen de déductions à partir d'axiomes, les inférences que l'on peut légitimement tirer des énoncés contenant savoir/ croire que; elle a enfin une portée explicative dès lors que ses axiomes reçoivent une interprétation dans le cadre de la philosophie de la connaissance (cf. Hendricks \& Symons 2009).

\subsection{Les inférences valides}

a) Différences entre savoir que et croire que :

Outre le fait que Je sais que ne supporte pas la négation, alors que Je crois que l'accepte sans difficulté :

(2a) ?* Je ne sais pas que $p$

(2b) Je ne crois pas que $p$.

p se laisse inférer des énoncés comportant «savoir que p », mais non de ceux qui contiennent «croire que $\mathrm{p} »$ :

(3) a. Il sait que $p$

$\left.\begin{array}{l}\text { a. Il ne sait pas que } \mathrm{p} \\ \text { c. Je sais que } \mathrm{p}\end{array}\right\} \Rightarrow p$

(4) a. Il croit que p

b. Il ne croit pas que $\mathrm{p}\} \neq>\mathrm{p}$.

c. Je crois que $p$

A partir des énoncés en savoir que, mais non de ceux en croire que, on infère «Je sais que $\mathrm{p} \gg$ :

$\left.\begin{array}{l}\text { (5) a. Il sait que } \mathrm{p} \\ \text { b. Il ne sait pas que } \mathrm{p}\end{array}\right\} \Rightarrow$ Je sais que $\mathrm{p}$

$\left.\begin{array}{l}\text { (6) a. Il croit que } \mathrm{p} \\ \text { b. Il ne croit pas que } \mathrm{p}\end{array}\right\} \neq>$ Je sais que $\mathrm{p}$.

b) Différences entre Je sais que (usage « direct ») et Il sait que (usage « oblique ${ }^{2}$ ) :

Aux exemples (5a), (5b) ci-dessus, on oppose :

(7) Je sais que $p \neq>$ Il sait que $p$.

\footnotetext{
${ }^{2}$ On reprend ici la terminologie de Martin (1987) qui ne parle d'usage direct que pour désigner les emplois à la première personne du présent de l'indicatif.
} 
Martin (1987 : 46) montre que de l'énonciation de «p », on infère «Je sais que p », mais non «Il sait que $\mathrm{p} »$ :

(8) a. $p \Rightarrow$ Je sais que $p$

b. $p \neq>$ Il sait que $p$.

«Je sais que $\mathrm{p}$ » est récursif, à la différence de «Il sait que $\mathrm{p} »$ :

(9) a. Je sais que $p \Rightarrow$ Je sais que je sais que $p$

b. Il sait que $\mathrm{p} \neq>$ Il sait qu'il sait que $\mathrm{p}$.

On peut, par exemple dire d'un chien «qu'il sait que son os est dans son panier » sans que cela implique que ce savoir soit conscient (i.e. qu'il sache qu'il le sait). En revanche, de «Il sait que $\mathrm{p}$ » on peut inférer que «Je sais qu'il sait que $\mathrm{p}$ », mais il est évidemment exclu de tirer «Il sait que je sais que $\mathrm{p} »$ de « Je sais que $\mathrm{p} »$ :

(10) a. Il sait que $\mathrm{p} \Rightarrow$ Je sais qu'il sait que $\mathrm{p}$

$b$. Je sais que $p \neq>$ Il sait que je sais que $p$.

On observe encore que si de «p » ou de «Il sait que $\mathrm{p} »$, on obtient «Je sais que $\mathrm{p} »$, on ne peut pour autant inférer «Je savais / saurai que $\mathrm{p} »$ :

(11) a. Pierre sait que $\mathrm{p}=>\left\{\begin{array}{l}\text { Je sais que } \mathrm{p} \\ \text { Je sais que }\end{array}\right.$ Je sais que Pierre sait que $\mathrm{p}$

b. Pierre sait que $p \neq>\{$ Je savais/saurai que $p$ Je savais/saurai que Pierre sait que $\mathrm{p}$.

Signalons enfin que si un individu sait que $\mathrm{p}$, il est impossible qu'un autre sache que non $\mathrm{p}$.

\subsection{Principes de logique épistémique}

Pour rendre compte de ces inférences, la logique épistémique stipule deux axiomes spécifiques, auxquels est généralement adjoint un principe concernant les rapports entre connaissance et assertion.

Les deux axiomes sont les suivants :

(12) Axiome de vérité (dit «axiome $\mathrm{T} »): \mathrm{Sxp} \Rightarrow \mathrm{p}$ [si un sujet $\mathrm{x}$ sait que $\mathrm{p}$, alors p est vraie]

(13) Axiome d'introspection positive (dit «axiome $4 »): \operatorname{Sxp} \Rightarrow \operatorname{SxSxp}$ [si x sait que p, alors $\mathrm{x}$ sait qu'il sait que $\mathrm{p}$.

L'adoption ou non de ce dernier axiome détermine le choix du système modal épistémique (selon la classification de Lemmon \& Scott 1977) : T ou S4. Ainsi l'exemple (9a) relève d'une logique de type $\mathbf{S 4}$ (qui admet à la fois les axiomes $\mathbf{T}$ et 4 ), mais (9b) du système $\mathbf{T}$ (qui admet l'axiome $\mathrm{T}$, mais refuse 4 ).

Pour dériver l'inférence (8a), il faut ajouter à ces axiomes un principe de philosophie du langage, présenté sous le nom de «principe d'implication de connaissance de l'assertion » par Williamson (2000) : 
(14) « On ne doit affirmer que p que si l'on sait que p» (cf. Engel $2007: 58$ ).

\subsection{Interprétation philosophique des axiomes épistémiques}

Ces deux axiomes de la logique épistémique ont été discutés dans le cadre de la philosophie de la connaissance. L'axiome $\mathrm{T}$ est généralement expliqué à partir de l'interprétation contemporaine de la conception platonicienne de la connaissance (Platon, Théétète 201) selon laquelle :

(15) S sait que $p$ si et seulement si

i) S croit que $p$ (condition de croyance)

ii) $p$ est vrai (condition de vérité)

iii) $p$ est justifié (condition de justification).

Cette conception, qui se retrouve également chez Kant (Logique IX), est résumée d'une formule par Engel (2007 : 25) :

(16) « savoir a une face interne, la croyance, et une face externe, la vérité. »

Si l'axiome T fait l'objet d'un consensus philosophique assez général, il n'en va pas de même pour l'axiome 4 (d'introspection positive), vis-à-vis duquel deux attitudes s'opposent nettement : l'Internalisme (représenté, entre autres, par le cartésianisme) admet ce principe et considère que «savoir c'est savoir que l'on sait» (Engel 2007 : 33). A l'inverse l'Externalisme le rejette et considère que le savoir peut fort bien être inconscient (c'est la position des théories de l'Inconscient, ainsi que de celles qui attribuent des connaissances «propositionnelles » aux animaux).

\subsection{Remarques linguistiques}

Le principe d'implication de connaissance de l'assertion peut se ramener à la Maxime de Qualité (Grice), et génère une implicature :

(17) En assertant $p$, le locuteur se présente comme sachant que $p:$ ASSERTxp $\Rightarrow \operatorname{Sxp}$ (où $x$ désigne le locuteur de l'assertion).

La conception objectiviste du savoir présupposée par l'axiome $\mathrm{T}$ ne paraît pas directement applicable à la sémantique de savoir que (cf. Vatrican 2012: 74). En effet, Brunot (1922) avait déjà souligné la compatibilité de savoir que avec des complétives exprimant un jugement subjectif, comme le montre aussi l'exemple (19) :

(18) «Les subordonnées qui suivent contiennent soit une certitude, soit une possibilité, une incertitude, un doute, une improbabilité : je sais [...] que la maladie était grave, qu'elle serait sans doute devenue mortelle. »(Brunot, 1922 : 528)

(19) «Vous saviez cependant que les Prussiens étaient les plus nombreux et que probablement ils seraient vainqueurs ! (Gaboriau, La corde au cou, Labyrinthes, éd. 2004 : 393).

Signalons que Beyssade (1994 : 89) propose, afin d'éviter tout recours à la notion de vérité objective, de remplacer l'axiome T, par la formule suivante : 
(20) « Un agent sait P s'il a un argument en faveur de P susceptible de convaincre les agents reconnus compétents. »

L'application de cette analyse à la sémantique linguistique se heurte cependant à ce que l'on peut considérer comme un véritable lieu commun du roman policier: l'enquêteur affirme « savoir » que tel individu est coupable, sans pour autant disposer de preuves recevables comme telles par un jury (représentant la communauté épistémique compétente). Ainsi Hercule Poirot :

(21) «C'est très différent de savoir qu'un homme est coupable et de prouver qu'il l'est. » (Christie, La mystérieuse affaire de Styles, trad. M. Logé, Le Livre de Poche, 1932 : 200).

Quant à l'axiome 4 (d'introspection positive), il ne peut convenir à l'analyse linguistique, pour la simple raison que le linguiste ne peut se permettre de convoquer, comme le logicien, tel ou tel système modal ( $\mathbf{T}$ ou $\mathbf{S 4}$ ) en fonction des énoncés à traiter, ou de choisir, comme le philosophe entre Externalisme et Internalisme : il doit proposer une analyse qui rende compte à la fois des inférences $(9 a)$ et $(9 b)$ et qui explique leur différence.

Enfin, en vertu de l'axiome $\mathrm{T}(\mathrm{Sxp} \Rightarrow \mathrm{p})$ et de l'implicature liée au principe d'implication de connaissance de l'assertion (ASSERTxp $\Rightarrow \operatorname{Sxp}$ ), «p » et «je sais que $\mathrm{p}$ », dès lors qu'elles sont assertées, sont équipollentes $(\operatorname{Sxp} \Leftrightarrow \mathrm{p}$ ). Dans une sémantique vériconditionnelle, elles sont donc tenues pour sémantiquement équivalentes (Descombes 2004 : 195-196), or on sait qu'elles ont des «fonctions communicatives fondamentalement différentes » (Vet 1994: 65). Une analyse linguistique adéquate de ces constructions doit permettre de rendre compte, outre des inférences légitimes que l'on peut tirer des énoncés qui les comprennent, des divers emplois de ces énoncés dans le discours.

\section{La solution proposée}

Nous proposons une nouvelle analyse de ces constructions dans le cadre de la Théorie Modulaire des Modalités (TMM, Gosselin 2010). Cette théorie se donne pour objet les modalités au sens large (au sens de Brunot 1922 et Bally 1932), qui englobent à la fois les valeurs modales des lexèmes (par exemple, la valeur axiologique de assassiner), les grammèmes marqueurs de modalité (comme les semi-auxiliaires et les adverbes modaux), mais aussi les modalités pragmatiquement inférées. Dans cette perspective, la modalité apparaît comme une catégorie fondamentalement hétérogène, présentant divers aspects syntaxiques, sémantiques, et pragmatiques. Alors que la sémantique formelle classique utilise un formalisme logique, qui traite chaque modalité comme un opérateur pourvu d'une syntaxe et d'une sémantique (cette syntaxe et cette sémantique étant empruntées aux logiques modales avec éventuellement des extensions spécifiques), la TMM met en œuvre, pour modéliser cette hétérogénéité, un formalisme informatique de type " orienté-objet », qui consiste à considérer chaque modalité comme un objet (noté $\bmod _{\mathrm{i}, \mathrm{j}, \mathrm{k}} \ldots$ ) auquel sont systématiquement associés neuf paramètres (ou attributs), susceptibles de prendre différentes valeurs.

\subsection{Les paramètres (ou attributs)}

Soit les neuf paramètres distingués (cf. Gosselin, 2010 : 57-142) :

Paramètres conceptuels [définissent des catégories et des valeurs modales] :

I : $\quad$ instance de validation [distingue les modalités objectives, subjectives ou institutionnelles]

D : direction d'ajustement [oppose les mod. à valeur descriptive aux mod. à valeur injonctive] [ces deux premiers paramètres définissent des catégories modales : aléthique, épistémique, appréciative, axiologique, déontique et boulique] 
F : force de la validation [précise la valeur à l'intérieur d'une catégorie, par ex. probable ou certain pour l'épistémique]

Paramètres fonctionnels [rendent compte du fonctionnement de la mod. dans l'énoncé] :

structuraux:

$\mathrm{N}$ : niveau dans la hiérarchie syntaxique

$\mathrm{P}$ : $\quad$ portée dans la structure logique

énonciatifs :

E : engagement du locuteur [marque le degré de prise en charge par le « locuteur de l'énoncé » $\left(1_{0}\right)^{3}$ ]

$\mathrm{R}$ : relativité [indique la relation éventuelle de la mod. à des éléments contextuels]

$\mathrm{T}: \quad$ temporalité [recouvre les caractéristiques temporelles et aspectuelles de la mod.]

Métaparamètre [indique si la valeur des autres paramètres a été obtenue par marquage linguistique ou par inférence] :

$\mathrm{M}$ : marquage.

Ainsi, le fait qu'une modalité (notée $\bmod _{\mathrm{i}}$ ) exprime un jugement subjectif, par exemple, sera noté comme suit : «I $\left(\bmod _{\mathrm{i}}\right)$ : subjectivité »; ce qui se lit : la valeur de l'instance de validation de la modalité $\bmod _{i}$ est la subjectivité (par opposition au réel ou à l'institution, qui caractérisent respectivement les modalités aléthique et déontique).

A tout énoncé se trouve associée une structure modale, qui comprend un certain nombre de modalités reliées entre elles par des relations logiques. Chacune de ces modalités est pourvue de ses neuf paramètres, auxquels sont assignées des valeurs particulières. Le calcul de cette structure modale et des valeurs des paramètres est effectué par un système de règles (dont l'architecture est modulaire). Dans ce système, les règles sont susceptibles de remplir deux rôles distincts : créer une modalité ou assigner une valeur à un paramètre.

Ces règles prennent pour entrées les marques linguistiques (on parle alors de « règles d'entrée »), ou des valeurs de paramètres déjà obtenues (« métarègles »).

Ces règles peuvent encore être de nature linguistique ou inférentielle. Dans ce dernier cas seulement, leur effet sera contextuellement annulable.

Modéliser un phénomène linguistique dans ce cadre, revient à :

a) identifier le type de structure modale en jeu : les modalités et leurs relations;

b) identifier les paramètres concernés;

c) formuler des règles pour calculer les structures modales et les valeurs des paramètres.

\subsection{Règles d'entrée : les constructions complétives}

On admet, à la suite de Pietrandrea (2010), et Pietrandrea \& Stathi (2010), que les « constructions » (au sens des grammaires de construction, cf. Goldberg 2010) peuvent servir d'entrées pour calculer des structures modales. Ainsi les constructions de type syntaxique «V que $\mathrm{p} »$ vont se voir associer systématiquement, au plan sémantique, une structure modale de la forme :

(22) Synt.: $\mathrm{V}$ que $\mathrm{P}$

Sem. : $\bmod _{\mathrm{i}}\left(\bmod _{\mathrm{j}}(\right.$ Pred $\left.(\arg )).\right)$

où $\bmod _{\mathrm{i}}$ est une modalité extrinsèque qui correspond au verbe de la principale, et $\bmod _{\mathrm{j}}$ une modalité intrinsèque au lexème prédicatif (Pred) de la complétive. Dans l'énoncé :

(23) Je doute qu'elle soit belle

\footnotetext{
${ }^{3}$ Nous adoptons la terminologie de la ScaPoLine, qui distingue, entre autres, le «locuteur de l'énoncé » $\left(l_{0}\right)$, responsable de l'énonciation, et le «locuteur textuel» (L), image du sujet parlant, qui, à la différence du précédent, transcende le hic et nunc énonciatif, cf. Nølke (2005), Kronning (ce volume, qui qualifie L de « locuteur du discours »).
} 
$\bmod _{\mathrm{i}}$ désigne la modalité épistémique de force négative marquée par le verbe douter, tandis que $\bmod _{\mathrm{j}}$ représente la modalité appréciative intrinsèquement associée au prédicat belle. En outre, cette analyse peut être étendue aux constructions du type «être Adj que p » (ex. «être certain que $\mathrm{p}$ » et «avoir SN que $\mathrm{p} »$ (ex. «avoir le sentiment que $\mathrm{p} »)$.

Cette structure renvoie à un frame correspondant à l'expression «d'un jugement $\left(\bmod _{\mathrm{i}}\right)$ sur un jugement $\left(\bmod _{\mathrm{j}}\right) »$. Ce jugement $\left(\bmod _{\mathrm{i}}\right)$ peut être simplement conçu (verbes d'attitude propositionnelle) ou énoncé (verbes dicendi); ce peut être la conception ou l'émission du jugement qui se trouve profilée (penser / dire que) ou sa réception (comprendre / apprendre / entendre que $)^{4}$. Le recours aulconcept de «construction » permet, outre la formulation d'une analyse très générale, la prise en compte de verbes qui sont normalement analysés comme intransitifs, par exemple trembler ou frémir dans «je tremble / frémis qu'il (ne) revienne ».

Dans ce cadre, le choix du verbe (ou des expressions adjectivales ou nominales) de la principale va servir à spécifier certaines valeurs des paramètres des modalités $\bmod _{\mathrm{i}}$ et $\bmod _{\mathrm{j}}$. En l'occurrence, on considère que savoir et croire contraignent les valeurs des paramètres conceptuels I, D et $\mathrm{F}$ de $\bmod _{\mathrm{i}}$ pour donner à cette modalité une valeur épistémique positive : I correspond à la subjectivité, $\mathrm{D}$ prend une valeur descriptive, la valeur de $\mathrm{F}$ est positive (alors qu'elle est négative avec douter). Mais ils affectent aussi la valeur du paramètre fonctionnel $\mathrm{E}$ de $\bmod _{j}$, et c'est en cela qu'ils diffèrent : savoir impose que le locuteur prenne en charge (accorde) $\bmod _{\mathrm{j}}$ (la validation du contenu de la complétive), alors que croire indique que le locuteur ne la prend pas en charge (il s'en dissocie), selon une classification des valeurs de E que l'on représente (à la suite de Nølke 1994, et Dendale \& Coltier 2005) comme suit :

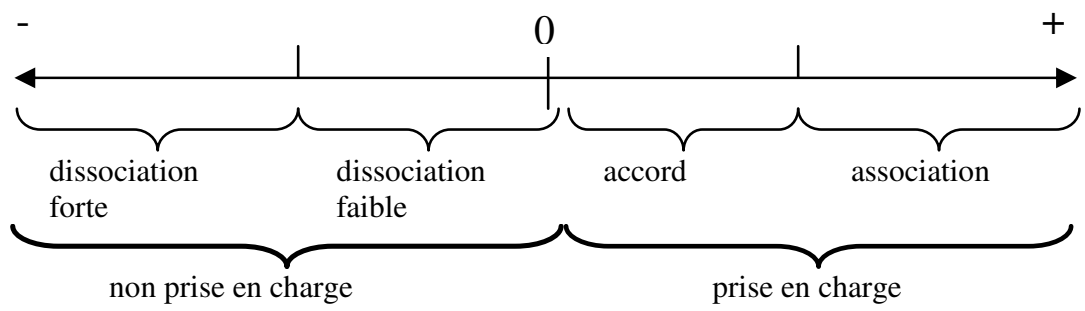

Figure 1: degrés de prise en charge

Soit, sous forme graphique :

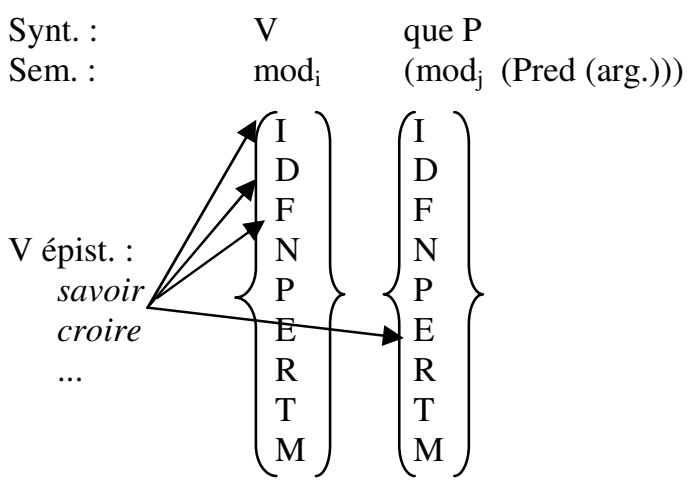

Figure 2 : contraintes exercées par les verbes épistémiques

\footnotetext{
${ }^{4}$ Selon Golberg (2010), une construction évoque un frame qui se trouve profilé (au sens de Langacker).
} 
Hors discours rapporté au style indirect, le locuteur dont il est question est le locuteur de l'énoncé ( $1_{0}$; voir note 3 ci-dessus). On obtient ainsi les structures modales :
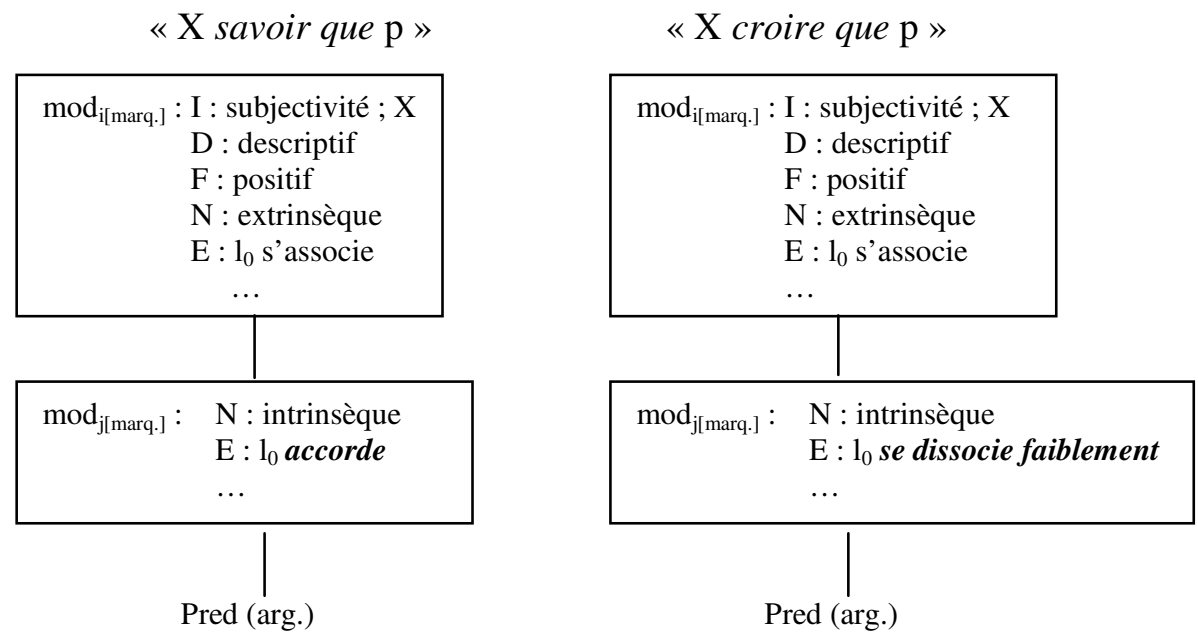

Figure 3 : structures modales résultant des règles d'entrée

Ce lien de prise en charge correspond à un traitement énonciatif de la factivité. Dire que savoir que est factif revient à dire qu'il indique que le locuteur de l'énoncé $\left(1_{0}\right)$ accorde la modalité intrinsèque au prédicat, autrement dit, qu'il présuppose le contenu de la complétive sans que cela implique aucunement qu'elle réfère à un fait présenté comme objectif.

\subsection{La métarègle de présomption de sincérité}

A ces règles d'entrée s'ajoute une métarègle inférentielle dite de "présomption de sincérité », qui conduit à construire une modalité subjective à valeur positive et à temps présent lorsque le locuteur (qu'il s'agisse du locuteur de l'énoncé $\left(l_{0}\right)$ ou de celui d'un discours rapporté) prend en charge (s'associe ou accorde) une modalité (paramètre E). En d'autres termes, on admet, par défaut, que si un locuteur prend en charge une modalité, c'est qu'il considère (en tant que locuteur textuel) que cette modalité est valide. L'application de cette métarègle à la structure associée à savoir que l'enrichit de la façon suivante : 


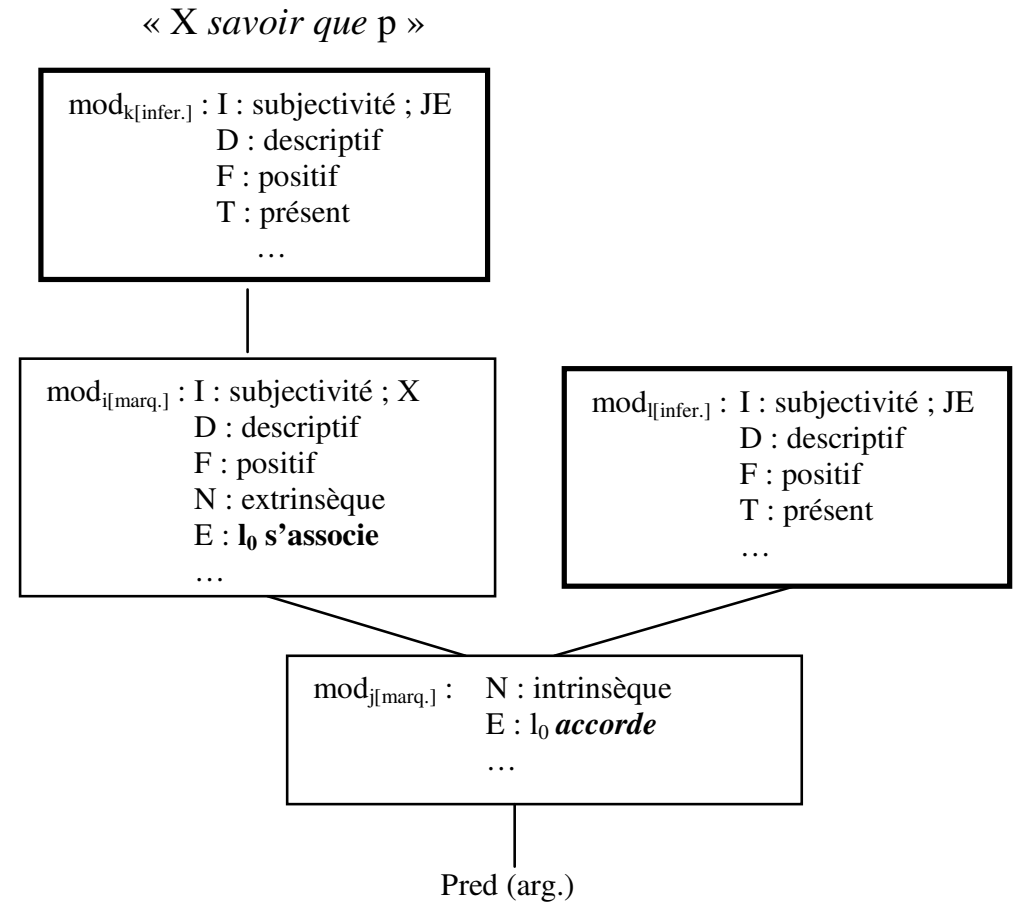

Figure 4 : structure modale enrichie par la métarègle

La structure enrichie associée à «X croire que $\mathrm{p}$ » est comparable, à la différence essentielle près qu'elle ne contient pas $\bmod _{1}: \operatorname{comme}_{0}$ ne prend pas en charge $\bmod _{\mathrm{j}}$, la métarègle de présomption de sincérité ne s'applique pas à cet endroit.

\subsection{Explication des inférences valides}

Ce dispositif permet d'expliquer assez simplement toutes les données présentées au $§ 2.1$. :

- « $\mathrm{p} »$ correspond à la prise en charge de la modalité intrinsèque à Pred (arg.) par $1_{0}$. En effet, lorsqu'un logicien pose « $\mathrm{p}$ », il ne veut pas seulement renvoyer au contenu propositionnel, comme pure représentation, mais à la validation de ce contenu, à sa présentation comme vrai. - Les modalités subjectives inférées sur la base de la prise en charge de la modalité par le locuteur $\left(\bmod _{\mathrm{k}}\right.$ et $\left.\bmod _{1}\right)$ sont exprimables au moyen de l'expression je sais que (première personne du présent), puisqu'elles articulent la subjectivité individuelle du locuteur (je) à la prise en charge par $1_{0}$ de la modalité sur laquelle elles portent (trait spécifique de savoir que).

Illustrons ce fonctionnement au moyen de l'analyse de l'exemple (11a). De «Pierre sait que $\mathrm{p}$ » (qui correspond à la fig.4), on infère $\mathrm{p}$ par la règle de présupposition liée à la factivité de savoir : le locuteur de l'énoncé accorde la modalité intrinsèque au prédicat de la complétive. De $\mathrm{p}$, ainsi présupposée, on dérive (par application de la métarègle de présomption de sincérité) que le locuteur textuel se constitue comme instance de validation d'une modalité épistémique positive $\left(\bmod _{1}\right)$ portant sur $\mathrm{p}$. Or cette modalité qui affecte $\mathrm{p}$ se laisse exprimer par la formule «Je sais que $\mathrm{p}$ ». De la même façon, on infère une modalité épistémique positive portant sur la modalité $\left(\bmod _{\mathrm{i}}\right)$ exprimée par Pierre sait que. Cette modalité inférée $\left(\bmod _{\mathrm{k}}\right)$ est exprimable à son tour par l'expression Je sais que (Pierre sait que). On voit simultanément pourquoi les inférences données en (11b) ainsi qu'en (7) ne sont pas valides : le temps des modalités inférées par la métarègle est nécessairement le présent et l'instance de validation correspond toujours à la première personne du singulier (qui représente le locuteur).

De même, on explique l'anomalie de «je ne sais pas / j'ignore que p », à partir de l'application de la métarègle : les verbes savoir et ignorer étant factifs, on dérive une mod, 
glosable par «je sais que $\mathrm{p}$ ». Seule une énonciation ouvertement insincère peut rendre ce type d'énoncé envisageable, comme dans cet extrait de roman policier, qui met en scène un assassin décidé à faire comme s'il ignorait qu'il vient de tuer sa femme, afin de détourner les soupçons (le locuteur textuel se trouve en quelque sorte dédoublé) :

(24) «Voyons! J'arrive de Nantes. J'ignore que ma femme est morte. » (Boileau-Narcejac, Celle qui n'était plus, Denoël, 1952 : 78).

\section{Extensions de l'analyse}

\subsection{La complétive contient une modalité extrinsèque}

Nous avons vu que, contrairement à ce que prévoit la conception classique de l'objectivité du savoir, il était tout à fait possible d'énoncer :

(25) Je sais qu'il va peut-être revenir.

Dans ces énoncés, la modalité associée à savoir ne porte plus directement sur la modalité intrinsèque au prédicat, mais sur une seconde modalité extrinsèque (notée $\left.\bmod _{\mathrm{i}}\right)$, qui vient s'intercaler entre les deux modalités précédemment repérées, dans la structure :

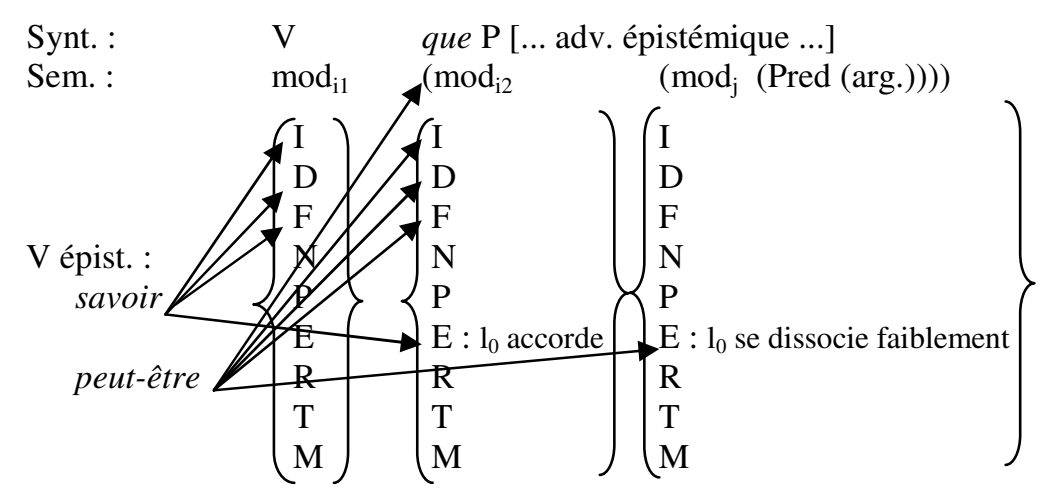

Figure 5 : contraintes exercées par le $\mathrm{V}$ et l'adverbe modal ${ }^{5}$

Ainsi la factivité de savoir que porte sur la seconde modalité extrinsèque $\left(\bmod _{\mathrm{i} 2}\right)$, qui est non factive. D'où la nécessité de distinguer entre factivité et factualité (catégories qui sont parfois assimilées) : la factualité correspond à la prise en charge par $1_{0}$ de la modalité intrinsèque au prédicat, tandis que la factivité d'un prédicat modal équivaut à la prise en charge (à valeur de présupposition) par $1_{0}$ de la modalité sur laquelle il porte directement. L'interprétation de (25) est factive, mais non factuelle.

\subsection{Factivité et discours rapporté}

Toute une littérature est consacrée, en sémantique formelle, à problématique de la « projection des présuppositions », qui consiste à prédire les présuppositions d'une phrase sur la base des présuppositions attachées à ses parties, ce qui revient - dans le cas qui nous occupe - à rendre compte du fait que les verbes épistémiques factifs conservent leur factivité dans certains contextes, alors qu'ils la perdent dans d'autres environnements.

Ainsi, savoir que reste factif lorsqu'il est régi par un verbe épistémique non factif, comme croire que ${ }^{6}$ (ex. 26a), mais il perd sa factivité sous la dépendance d'un verbe dicendi

\footnotetext{
${ }^{5}$ On ne représente par les contraintes exercées sur N, P et T, par souci de lisibilité.
} 
non factif (Karttunen 1973, Mørdrup 1975) (ex. 26b), sauf si ce verbe est mis à la forme négative (Heim 1992), à condition toutefois que cette dernière soit interprétée comme négation descriptive (ne pas dire équivalant alors à taire, ex. 26c) :

(26) a. Jean a cru que Marie savait que Pierre lui était infidèle (factif)

b. Jean m'a dit que Marie savait que Pierre lui était infidèle (non factif)

c. Jean ne m'a pas dit que Marie savait que Pierre lui était infidèle (factif).

Ces phénomènes s'expliquent aisément dès lors que l'on admet que savoir que indique que la modalité sur laquelle il porte est accordée par le locuteur, et que la factivité correspond à la prise en charge de cette modalité par $\mathrm{l}_{0}$. Car dans le cas des verbes dicendi, c'est le locuteur du discours rapporté (et non plus $1_{0}$ ) qui prend en charge (accorde) la modalité intrinsèque au prédicat de la complétive. Cette analyse se trouve confirmée par le fait que lorsqu'il est dit que le discours n'a pas été rapporté, pris en charge par un autre locuteur (ex. 26c), la construction reste factive. Ce n'est donc pas savoir que qui change de valeur dans ce contexte, mais uniquement le locuteur responsable de la prise en charge. On considère qu'un verbe factif ne fait l'objet d'une lecture factive que si le locuteur responsable de la prise en charge est le locuteur de l'énoncé $\left(l_{0}\right)$.

\subsection{Prédicats contrefactifs}

A côté des prédicats factifs et non factifs, il existe des prédicats semi-factifs (Kreutz 1998, Korzen 2001) et contrefactifs (Meinunger 2007 : 161), qui indiquent que le locuteur se dissocie fortement de la modalité sur laquelle ils portent. Ainsi les verbes s'imaginer que et se figurer que expriment-ils à la fois une modalité épistémique de force positive (comparable à croire que) et le fait que le locuteur refuse de prendre en charge la modalité qu'ils affectent. Or à cette dissociation forte est associée une métarègle qui fait le pendant exact de la présomption de sincérité : si le locuteur se dissocie fortement de la modalité, c'est qu'il doute. De là, la création d'une modalité subjective individuelle à valeur négative et à temps présent portant sur la modalité dont le locuteur (qu'il s'agisse du locuteur de l'énoncé, $1_{0}$, ou de celui d'un discours rapporté) se dissocie fortement. Soit la structure associée à s'imaginer que :

\footnotetext{
${ }^{6}$ Certes, savoir que perd sa factivité dans «je crois savoir que $\mathrm{p} »$, mais il s'agit alors d'une autre construction, qui ne peut être assimilée à «?? je crois que je sais que $\mathrm{p}$ ».
} 
«X s'imaginer que »

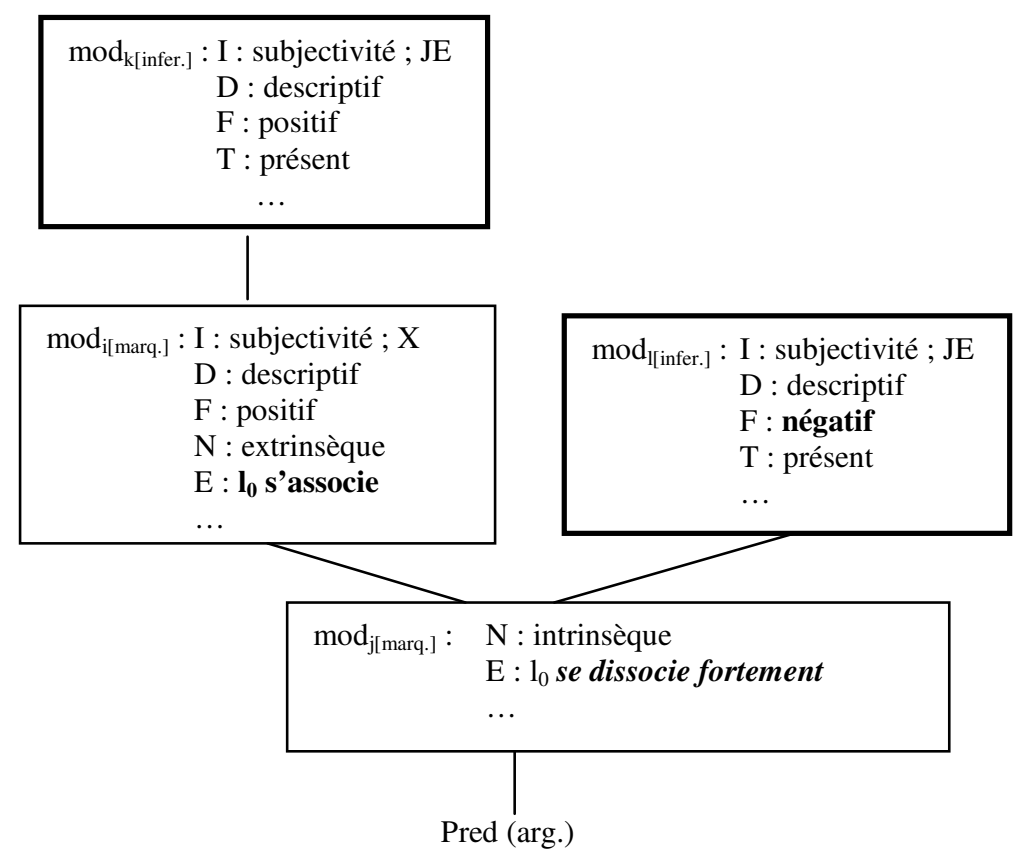

Figure 6 : structure modale enrichie par la métarègle

Cette structure rend compte des inférences valides :

(27)

Pierre s'imagine que $\mathrm{p} \Rightarrow\left\{\begin{array}{l}\text { non } \mathrm{p} \\ \text { Pierre croit que } \mathrm{p} \\ \text { j'exclus que } \mathrm{p}\end{array}\right.$

ainsi que des anomalies :

(28) a. ?* Je m'imagine que non $\mathrm{p}$

b. ?* Je m'imagine que je sais que non $\mathrm{p}$.

(28a) n'est guère interprétable (sauf si l'on interprète s'imaginer dans le sens de «se construire une représentation mentale fictive »), car cette phrase exprime à la fois «je crois que $\mathrm{p} »\left(\bmod _{\mathrm{i}}\right)$ et «j'exclus que $\mathrm{p} »\left(\bmod _{1}\right)$.

Tout comme la factivité de savoir que - et pour les mêmes raisons - la contrefactivité de s'imaginer que se trouve suspendue par le discours rapporté (sauf avec la négation descriptive) :

(29) a. Pierre dit que Jean s'imagine que Marie le trompe ( $\neq>$ Marie ne le trompe pas)

b. Pierre ne m'a pas dit que Jean s'imaginait que Marie le trompait $(\Rightarrow$ Marie ne le trompe pas).

De même qu'il faut distinguer la factivité de la factualité, il est nécessaire de dissocier le contrefactif du contrefactuel, pour rendre compte du fait que lorsqu'un verbe contrefactif porte sur une modalité extrinsèque, cela n'implique pas toujours l'irréalité du fait exprimé. Exemple :

(30) Pierre s'imagine qu'il va forcément / nécessairement gagner la course. 
Ce qui est rejeté dans cet exemple, c'est uniquement l'inéluctabilité (comme nécessité aléthique prospective) de la victoire, selon une structure comparable à celle de la Figure 5.

\section{Modalisation}

Il nous paraît indispensable de distinguer la «modalité », comme catégorie syntacticosémantique, de la «modalisation », comme procédé consistant à employer une modalité particulière à des fins pragmatiques, illocutoires et/ou perlocutoires, dans une situation de discours donnée. Etant donné la très grande variété des usages discursifs des modalités épistémiques, nous ne pouvons en donner ici qu'un aperçu extrêmement limité.

Dès lors que l'on quitte le cadre vériconditionnel de la logique épistémique pour une approche énonciative de la sémantique des jugements épistémiques, on n'a plus à considérer « $\mathrm{p}$ » et «je sais que $\mathrm{p} »$ comme des formulations équivalentes (parce qu'équipollentes) : dans le premier cas, le locuteur asserte $\mathrm{p}$ : il s'associe à la modalité intrinsèque au prédicat; dans le second il présuppose $\mathrm{p}$ : il accorde la modalité intrinsèque. Cela explique certains emplois comme le je sais des adolescents (cf. Franckel \& Lebaud 1990) qui veulent signifier à leurs parents que leur discours est non informatif (parce que p est présupposée), ou le fait que $\mathrm{p}$ ne puisse faire l'objet d'une discussion (p étant déjà admise) ${ }^{7}$. Cela explique aussi pourquoi je sais, par opposition à je crois ne peut constituer une réponse à une interrogation totale (cf. Vet 1994 : 60), le locuteur ne pouvant accorder une proposition dont la valeur de vérité reste inconnue :

(31) - Marie est-elle là ?

- Je crois / ??Je sais.

C'est ce que confirme le fait que j'en doute (non factif) constitue une réponse possible, alors que je m'en doute (factif) n'en est pas une ${ }^{8}$.

On remarque, en outre, que la prédiction des inférences légitimes permet aussi d'expliquer certains usages en discours. Ainsi, on rencontre souvent les expressions «tout le monde sait que $\mathrm{p}$ », «qui ne sait que $\mathrm{p}$ ? » ou «chacun sait que $\mathrm{p}$ », ou encore «nul n'ignore que $\mathrm{p}$ », qui servent à donner à l'énoncé valeur d'argument d'autorité (incontestable a priori). Mais on ne trouve pas, dans ce type d'emploi, «les gens savent que $\mathrm{p}$ ». Cela s'explique si l'on reconnait que toute expression du type «X sait que $\mathrm{p}$ » implique «je sais que $\mathrm{p}$ ». De «tout le monde sait que $\mathrm{p}$ », on infère $\mathrm{p}$ (factivité); et de $\mathrm{p}$, «je sais que $\mathrm{p} »$ (métarègle), ce qui ne pose aucun problème puisque le locuteur est inclus dans tout le monde. De «les gens savent que $\mathrm{p}$ », on infèrerait, de la même manière, $\mathrm{p}$, puis «je sais que $\mathrm{p}$ », ce qui, cette fois poserait problème puisque le locuteur (je) n'est, comme le montre Schnedecker (2012), pas inclus dans les gens (d'où les énoncés du type : les gens sont bêtes / bizarres, etc.). En vertu de la loi d'exhaustivité (Ducrot 1972) ou de la maxime de quantité de Grice, on est même conduit à penser que si le locuteur dit «les gens + Prédicat », c'est qu'il considère que le prédicat ne s'applique pas au complémentaire de l'ensemble dénoté par les gens, c'est-à-dire à lui-même, ce qui est contradictoire avec l'implication de «je sais que $\mathrm{p}$ » par «les gens savent que $\mathrm{p}$ ». Seule l'adjonction d'un marqueur à valeur confirmative peut suspendre l'implicature et rendre l'expression utilisable dans cet emploi («les gens savent bien / effectivement / naturellement que $\mathrm{p} \gg)$.

\footnotetext{
${ }^{7}$ D'autres emplois discursifs sont évoqués dans Vet (1994) et Vatrican (2012: 81).

${ }^{8}$ Je l'ignore constituerait un contre-exemple si ce tour ne renvoyait à «j'ignore si $\mathrm{p}$ » plutôt qu'à «j'ignore que $\mathrm{p} »$.
} 


\section{Conclusion}

L'analyse linguistique des verbes épistémiques, proposée comme alternative à celle de la logique épistémique, nous a conduit à avancer ou à affermir certaines distinctions conceptuelles que nous résumons pour conclure.

Tout d'abord, il est apparu qu'il ne pouvait y avoir correspondance exacte entre une conception philosophique du savoir (ou de la connaissance) et la sémantique de «savoir que $\mathrm{p}$ ». Si l'on peut, sur la base d'arguments philosophiques, défendre l'objectivité du savoir, ou au moins son caractère de certitude intersubjective, on a vu que «savoir que $\mathrm{p}$ » s'accommode parfaitement de jugements de (faible) probabilité subjective (ex. je sais qu'elle est peut-être là). C'est pourquoi les verbes épistémiques relèvent d'analyses proprement linguistiques.

Cependant ces verbes épistémiques ne peuvent être étudiés indépendamment des constructions dans lesquelles ils apparaissent. On sait, par exemple, que la sémantique de savoir que est différente de celles, toutes distinctes les unes des autres, de savoir si, savoir $S N$, savoir Vinf, savoir où / qui / quand / comment... C'est pourquoi nous avons assigné une structure modale aux constructions complétives (V que p), considérant que le choix du verbe épistémique venait spécifier la valeur de certains paramètres des modalités de cette structure.

Plus précisément, on considère qu'un verbe épistémique, dans une construction complétive, marque à la fois le degré de la croyance exprimée et la prise en charge de la modalité intrinsèque au prédicat sur lequel porte cette croyance.

Cette distinction suppose aussi que l'on dissocie le locuteur textuel, susceptible d'être présenté comme ayant des croyances et divers états mentaux, du « locuteur de l'énoncé » $\left(1_{0}\right)$, «qui, n'ayant d'existence que dans le hic et nunc énonciatif, assume la responsabilité (modale) de l'énoncé » (Kronning, 2009: 101). Un tour comme «je crois que $\mathrm{p} »$ montre que le locuteur textuel (image de l'être du monde) adhère à la proposition (la force $\mathrm{F}$ de $\bmod _{\mathrm{i}}$ est positive), mais que le locuteur de l'énoncé ne la prend pas en charge (il se dissocie faiblement de $\left.\bmod _{\mathrm{j}}\right) ;$ d'où l'effet, en discours, de modalisation atténuative.

On considère, par ailleurs, qu'un verbe factif ne donne pas nécessairement lieu à une interprétation factive. Un verbe factif est un verbe qui indique que le locuteur prend en charge la modalité sur laquelle porte ce verbe. Si le locuteur en question est le locuteur de l'énoncé $\left(1_{0}\right)$, la lecture sera factive («il sait que $\left.\mathrm{p} »\right)$, tandis que s'il s'agit du locuteur d'un discours rapporté, la lecture sera non factive («il dit qu'il sait que $\mathrm{p} »)$, à moins que le verbe introducteur du discours rapporté ne soit lui-même factif («il nous a révélé qu'il savait que $\mathrm{p} »)$.

De plus, la factivité n'implique pas toujours la factualité. Ainsi, lorsqu'un verbe factif, en lecture factive, porte sur une autre modalité extrinsèque à valeur de probabilité faible, l'interprétation sera non factuelle, quoique factive (je sais qu'il est peut-être là).

Les mêmes distinctions s'appliquent mutatis mutandis à la contrefactivité («s'imaginer que $\mathrm{p} »)$. Un verbe contrefactif ne donne lieu à une lecture contrefactive que si c'est le locuteur de l'énoncé $\left(l_{0}\right)$ et non celui d'un discours rapporté qui se présente comme refusant de prendre en charge la modalité sur laquelle porte le verbe en question. De même, la contrefactivité n'implique pas nécessairement la contrefactualité, en particulier lorsque le verbe contrefactif, en lecture contrefactive, porte sur une autre modalité extrinsèque exprimant la nécessité (il s'imagine qu'il va nécessairement réussir).

Enfin, nous tenons à dissocier la modalité, comme catégorie syntactico-sémantique, de la modalisation comme procédé sémantico-pragmatique consistant à utiliser des modalités à des fins illocutoires et perlocutoires. L'explicitation du rôle des modalités dans la détermination, en situation, de la force illocutoire et des effets perlocutoires relève d'une théorie sémanticopragmatique de la modalisation dont la sémantique des modalités doit fournir les bases linguistiques. 


\section{Bibliographie}

BALly Ch. (1932), Linguistique générale et linguistique française, Paris : Leroux.

BARBET C. \& SAUSSURE, L. DE (éds) (2012), Langue française 173 : Modalité et évidentialité en français, Paris : Larousse-Armand Colin.

BEYSSADE C. (1994), Les modalités épistémiques dans un système multi-agent : contribution à l'étude linguistique de l'opacité et des présuppositions, Thèse de l'Université de Caen.

BRUNOT F. (1922), La pensée et la langue, Paris : Masson.

DENDALE P. \& COLTIER D. (2005), «La notion de prise en charge ou de responsabilité dans la théorie scandinave de la polyphonie linguistique », in J. Bres et al. (éds), Dialogisme et polyphonie. Approches linguistiques, Bruxelles : De Boeck-Duculot, 125-140.

DESCOMBES V. (2004), Le complément de sujet, Paris : Gallimard.

DuCROT O. (1972), Dire et ne pas dire, Paris : Hermann.

ENGEL P. (2007), Va savoir! De la connaissance en général, Paris : Hermann.

FRANCKEL J.-J. \& LEBAUD D. (1990), Les figures du sujet, Gap : Ophrys.

GARDIES J.-L. (1979), Essai sur la logique des modalités, Paris : PUF.

GoldBerg A. (2010), "Verbs, Constructions and Semantic Frames", in M. Rappaport Hovav, E. Doron \& I. Sichel (eds), Lexical Semantics, Syntax and Event Structure, Oxford: U.P, 39-58.

Gosselin L. (2010), Les modalités en français. La validation des représentations, Amsterdam-New York : Rodopi.

HeIM I. (1992), "Presupposition Projection and the Semantics of Attitude Verbs", Journal of Semantics 9, 183-221.

HendRICKS V. \& SyMOnS J. (2009), "Epistemic Logic", in E. N. Zalta (ed.), The Stanford Encyclopedia of Philosophy, http://plato.stanford.edu/archives/spr2009/entries/logicepistemic.

HinTIKKA J. (1962), Knowledge and Belief. An Introduction to the Logic of the two Notions, Ithaca, New York: Cornell U.P.

KARTTUnen L. (1973), "Presuppositions of Compound Sentences", Linguistic Inquiry IV, 169-193.

KORZEN H. (2001), «Factivité, semi-factivité et assertion : le cas des verbes savoir, ignorer, oublier et cacher », in $\mathrm{H}$. Kronning et al. (éds), Langage et référence, Uppsala : Acta Universitatis Upsaliensis, 323-333.

KreUtz Ph. (1998), «Une typologie des prédicats factifs », Le Français moderne 66, 141181.

KRONNING H. (2009), "Polyphonie, constructions conditionnelles et discours rapporté », Langue Française 164, 97-111.

LEMMON E. J. \& SCOTT D. (1977), An introduction to modal logic, Oxford: Blackwell.

LENZEN W. (2004), "Epistemic Logic", in I. Niinihuoto, M. Sintonen \& J. Volenski (eds), Handbook of Epistemology, Dordrecht: Kluwer, 963-984.

MARTIN, R. (1987), Langage et croyance, Bruxelles : Mardaga.

MeINunger A. (2007), "In the Mood of Desire and Hope: Remarks on the German Subjunctive, the Verb second Phenomenon, the Nature of Volitional Predicates and Speculations on Illocution", Cahiers Chronos 17, 155-176.

MøRDRUP O. (1975), «Présuppositions, implications et verbes français », Revue Romane 10, 125-157.

NøLKE H. (1994), Linguistique modulaire : de la forme au sens, Louvain, Paris : Peeters. 
NøLKE H. (2005) «Le locuteur comme constructeur de sens », in J. Bres et al. (éds), Dialogisme et polyphonie. Approches linguistiques, Bruxelles: De Boeck-Duculot, 111124.

PIETRANDREA P. (2010), Constructions grammaticales et discours, Dossier d'HDR, Université Paris 3.

PietrandReA P. \& StATHI K. (2010), "What counts as an evidential unit? The case of evidential complex constructions in Italian and Modern Greek", STUF-Sprachtypologie und Universalienforschung, 63-4, 333-344.

SCHNEDECKER C. (2012), «Tout le monde, tous, (tous) les gens : relations sémantiques entre des expressions dénotant la totalité /+hum/», in N. Le Querler, F. Neveu \& E. Roussel (éds), Relations, connexions, dépendances, P.U. Rennes, 127-155.

VAtrican, A. (2012), «Savoir que et la notion de présupposition », Langages 186, 69-84.

VET C. (1994), « Savoir et croire », Langue Française 102, 56-68.

WILLIAMSON T. (2000), Knowledge and its limits, Oxford: U.P. 